\title{
Characterisation of fibrosis in chemically-induced rat mammary carcinomas using multi-modal endogenous contrast MRI on a $1.5 T$ clinical platform
}

\author{
Neil P. Jerome ${ }^{1}$ - Jessica K. R. Boult ${ }^{1}$ - Matthew R. Orton ${ }^{1}$ - James A. d'Arcy ${ }^{1}$. \\ Ashutosh Nerurkar ${ }^{2}$ - Martin O. Leach ${ }^{1}$ - Dow-Mu Koh ${ }^{1,3}$ - David J. Collins ${ }^{1}$. \\ Simon P. Robinson ${ }^{1}$
}

Received: 19 January 2017 / Revised: 25 August 2017 / Accepted: 14 September 2017 / Published online: 16 October 2017

(C) The Author(s) 2017. This article is an open access publication

\begin{abstract}
Objectives To determine the ability of multi-parametric, endogenous contrast MRI to detect and quantify fibrosis in a chemically-induced rat model of mammary carcinoma.

Methods Female Sprague-Dawley rats $(\mathrm{n}=18)$ were administered with $N$-methyl- $N$-nitrosourea; resulting mammary carcinomas underwent nine-b-value diffusion-weighted (DWI), ultrashort-echo (UTE) and magnetisation transfer (MT) magnetic resonance imaging (MRI) on a clinical 1.5T platform, and associated quantitative MR parameters were calculated. Excised tumours were histologically assessed for degree of necrosis, collagen, hypoxia and microvessel density. Significance level adjusted for multiple comparisons was $\mathrm{p}=0.0125$.

Results Significant correlations were found between MT parameters and degree of picrosirius red staining $(r>0.85, p<$ 0.0002 for $\mathrm{k}_{\mathrm{a}}$ and $\delta, \mathrm{r}<-0.75, \mathrm{p}<0.001$ for $\mathrm{T}_{1}$ and $\mathrm{T}_{1 \mathrm{~s}}$, Pearson), indicating that MT is sensitive to collagen content in mammary carcinoma. Picrosirius red also correlated with
\end{abstract}

Neil P. Jerome-and Jessica K. R. Boult contributed equally to this work

David J. Collins and Simon P. Robinson contributed equally to this work

Electronic supplementary material The online version of this article (https://doi.org/10.1007/s00330-017-5083-6) contains supplementary material, which is available to authorized users.

Simon P. Robinson

Simon.Robinson@icr.ac.uk

CR-UK Cancer Imaging Centre, Division of Radiotherapy and Imaging, The Institute of Cancer Research, London SM2 5NG, UK

2 Department of Histopathology, Royal Marsden NHS Foundation Trust, London SW3 6JJ, UK

3 Department of Radiology, Royal Marsden NHS Foundation Trust, London SM2 5PT, UK the DWI parameter $\mathrm{fD}^{*}(\mathrm{r}=0.801, \mathrm{p}=0.0004)$ and conventional gradient-echo $\mathrm{T}_{2} *(\mathrm{r}=-0.660, \mathrm{p}=0.0055)$. Percentage necrosis correlated moderately with ultrashort/conventional-echo signal ratio $(r=0.620, p=0.0105)$. Pimonidazole adduct (hypoxia) and CD31 (microvessel density) staining did not correlate with any MR parameter assessed.

Conclusions Magnetisation transfer MRI successfully detects collagen content in mammary carcinoma, supporting inclusion of MT imaging to identify fibrosis, a prognostic marker, in clinical breast MRI examinations.

Key Points

- Magnetisation transfer imaging is sensitive to collagen content in mammary carcinoma.

- Magnetisation transfer imaging to detect fibrosis in mammary carcinoma fibrosis is feasible.

- IVIM diffusion does not correlate with microvessel density in preclinical mammary carcinoma.

Keywords Functional magnetic resonance imaging · Magnetisation transfer contrast imaging · Fibrosis · Mammary neoplasms · Mammary carcinoma, animal

$\begin{array}{ll}\text { Abbreviations } \\ \text { ADC } & \text { Apparent diffusion coefficient } \\ \text { ARRIVE } & \text { Animal Research Reporting In Vivo Experiments } \\ \text { CoV } & \text { Coefficient of variation } \\ \text { DCE } & \text { Dynamic contrast-enhanced } \\ \text { DWI } & \text { Diffusion-weighted imaging } \\ \text { EPI } & \text { Echo-planar imaging } \\ \text { FID } & \text { Free induction decay } \\ \text { FITC } & \text { Fluorescein isothiocyanate } \\ \text { GRE } & \text { Gradient echo (also mGRE multiple gradient } \\ & \text { echo) }\end{array}$




$\begin{array}{ll}\text { H\&E } & \text { Haematoxylin and eosin } \\ \text { IVIM } & \text { Intravoxel incoherent motion } \\ \text { LOOCV } & \text { Leave-one-out cross-validation } \\ \text { LV } & \text { Latent variable } \\ \text { LR } & \text { Linear regression } \\ \text { MCMC } & \text { Markov chain Monte Carlo } \\ \text { MNU } & N \text {-methyl- } N \text {-nitrosourea } \\ \text { MRI } & \text { Magnetic resonance imaging } \\ \text { MT } & \text { Magnetisation transfer } \\ \text { MTR } & \text { Magnetisation transfer ratio } \\ \text { MVD } & \text { Microvessel density } \\ \text { NRMSE } & \text { Normalised root mean square error } \\ \text { PLSR } & \text { Partial least squares regression } \\ \text { ROI } & \text { Region of interest } \\ \text { TMJ } & \text { Temporomandibular joint } \\ \text { UTE } & \text { Ultrashort echo time }\end{array}$

\section{Introduction}

Breast cancer development and growth is strongly influenced by the crosstalk of tumour cells with the surrounding extracellular matrix/stroma [1-3]. The stroma can make up a significant proportion of a breast carcinoma [4], and differs from normal stroma, bearing closer resemblance to granulation tissue and wound healing, with a high number of fibroblasts, deposition of type I collagen and fibrin, and the infiltration of inflammatory cells [5]. The presence of a fibrotic focus, a central scar-like area within a carcinoma that represents a focus of exaggerated reactive tumour stromal formation, was first proposed as an indicator of increased tumour aggressiveness in invasive ductal breast cancer by Hasabe et al. [6], and has since been linked to early disease relapse, lymph node and osteolytic bone metastasis, and reduced long-term survival [7-9]. Hypoxia has also been associated with the formation of fibrotic foci [5].

Advanced MRI techniques provide a means of defining non-invasive quantitative biomarkers to inform on biologically relevant structure-function relationships in tumours, thereby enabling an understanding of their behaviour and heterogeneous distribution [10]. Imaging biomarkers for assessing tumour pathophysiology require evaluation before being routinely deployed in clinical trials; in particular, imagingpathology correlation, and thus whether the imaging biomarker reflects underlying pathology is important to establish, but can often only meaningfully be studied in animal models [11].

The newly generated tumour stroma shows similarities with granulation tissue and subsequent scar formation in wound healing and differs from the normal stroma by an increased number of fibroblasts, enhanced capillary density, deposition of type I collagen and fibrin and the presence of inflammatory cells.
Several MRI biomarkers have the potential to detect breast cancer fibrosis. The fibrous nature of collagen may increase the non-monoexponential contribution to the diffusion-weighted MRI (DWI) signal, arising from the propensity of water molecules to diffuse along the fibres, combined with reduced diffusivity from encountering more barriers to random diffusion, compared to surrounding tissue [12-14]. Increased macromolecular collagen fibre content may also yield a greater destruction of signal arising from MT MRI from off-resonance saturation [15], and the short-lived signal of collagen $\left(\mathrm{T}_{2} * \sim 500 \mu \mathrm{s}\right)$ may be detectable with ultrashort-echo time (UTE) sequences [16]. Dynamic contrast-enhanced (DCE) MRI remains a standard technique used in breast cancer MRI protocols and may be suitable for fibrosis detection in some tissues [17], but the use of contrast adds complexity to clinical studies and can be contraindicated in certain patients.

This study aims to determine the ability of multi-parametric MRI incorporating several endogenous contrast mechanisms, such as DWI, MT-MRI and UTE-MRI, performed on a clinical imaging platform, to detect and quantify fibrosis in a chemically-induced rat model of mammary carcinoma previously shown to produce heterogeneous tumours with a range of fibrosis severity [18].

\section{Materials and methods}

\section{Animal procedures}

This study was performed in accordance with the local ethics review panel, United Kingdom National Cancer Research Institute guidelines for animal welfare in cancer research, and the ARRIVE (Animal Research: Reporting In Vivo Experiments) guidelines [19, 20]. Female Sprague-Dawley rats (200-250 g, n=18; Charles River, Margate, UK) were injected with $37.5 \mathrm{mg} \cdot \mathrm{kg}^{-1}$ of refrigerated $N$-methyl $-N$ nitrosourea (MNU, Sigma-Aldrich, Poole, UK) intraperitoneally, resulting in tumours that spontaneously developed at various sites associated within the mammary fat-pad [18]. Tumour formation was detected by palpation and growth was monitored by calliper measurement; animals were imaged when tumours reached approximately $3 \mathrm{~cm}^{3}$ (using ellipsoid volume formula, $(\pi / 6) \times \mathrm{L} \times \mathrm{W} \times \mathrm{D})$.

Animals were anaesthetised using a $4 \mathrm{ml} . \mathrm{kg}^{-1}$ intraperitoneal injection of fentanyl citrate $\left(0.315 \mathrm{mg} \cdot \mathrm{ml}^{-1}\right)$ plus fluanisone $\left(10 \mathrm{mg} \cdot \mathrm{ml}^{-1}\right.$ (Hypnorm; Janssen Pharmaceutical Ltd., High Wycombe, UK)), midazolam (5 mg. $\mathrm{ml}^{-1}$ (Hypnovel; Roche)), and water (1:1:2). Prior to imaging, an intraperitoneal injection of $60 \mathrm{mg} \cdot \mathrm{kg}^{-1}$ pimonidazole (Hypoxyprobe, Burlington, VT, USA) in phosphate- buffered saline was given, in preparation for subsequent histological staining for hypoxia. 


\section{Magnetic resonance imaging}

MR imaging was performed on a MAGNETOM Avanto $1.5 \mathrm{~T}$ clinical scanner (Siemens Healthcare, Erlangen, Germany), to validate clinical sequences and support methodological transfer. For MRI, the animal was secured supine using an insulating vacuum beanbag to both retain body heat and prevent excessive movement. The animal was placed with the tumour centred on top of a small-loop temporomandibular joint (TMJ) coil, itself centred within the multi-element head receiver coil [21]. Elements of the head coil array were used in parallel with the small-loop coil during all acquisitions. Scans were performed in the coronal plane, with full tumour coverage. Morphological $\mathrm{T}_{2}$-weighted fast spin-echo images were obtained for anatomical localisation. Diffusion-weighted MRI (DWI), ultrashort-echo time (UTE) MRI and magnetisation transfer (MT) data were acquired centred on the lesion.

UTE data were acquired with a prototype threedimensional (3D) multiple gradient echo (mGRE) sequence with $1.1 \mathrm{~mm}$ isotropic resolution; the first echo acquired was on the free induction decay (FID) immediately following the read pulse, followed by four regular gradient echoes. This acquisition was repeated in order to acquire four ultrashort-echo times (70-560 $\mu \mathrm{s})$. DWI was based on a clinical patient protocol (nine b-values, $0-800 \mathrm{~mm}^{-2}$ s; see Table 1) acquired in free-breathing using a fat-suppressed two-dimensional (2D) single-shot prototype EPI sequence. MT data were acquired as a series of matched 3D GRE acquisitions, with 1.0-mm isotropic voxels, and two flip angles with/without an MT pulse set at $1.5 \mathrm{kHz}$ offset.
Detailed sequence parameters are given in Table 1, and were adapted from clinical imaging sequences; the total acquisition time was approximately 1 hour.

\section{MR image analysis}

MRI analysis was performed using proprietary software (ADEPT, The Institute of Cancer Research, London, UK). All MR images were reviewed, and regions of interest (ROIs) were independently drawn by two observers, MR scientists (NPJ and DJC) with 5 and 32 years' experience, respectively, in conducting preclinical MR studies. Repeatability of ROI delineation was assessed using the Sørenson-Dice similarity coefficient. Each ROI was drawn around the tumour on the imaging slice that macroscopically matched the histological section stained, MR parameters calculated on a voxel-by-voxel basis, and reported as the average value for repeated ROI median values per slice analysed together with calculation of repeat-measures coefficient of variation $(\mathrm{CoV})$.

For DWI analysis, the perfusion-insensitive apparent diffusion coefficient (ADC) was estimated using images for $b=200$ $\mathrm{mm}^{-2} \mathrm{~s}$ and above [22], with a single-exponential model (Eq. 1). All b-values were used for intravoxel incoherent motion (IVIM) fitting using a bi-exponential model (Eq. 2) to simultaneously derive estimates of pseudodiffusion fraction (f), pseudodiffusion coefficient (D*) and tissue diffusivity (D). The compound parameter $\mathrm{fD}^{*}$ was also calculated. Initial estimate values for IVIM fitting were found using the segmented approach [22], by estimating D using a monoexponential fit of images with $\mathrm{b}=200 \mathrm{~mm}^{-2} \mathrm{~s}$ and above (as per ADC) and f from

Table 1 MR imaging parameters for anatomical imaging $\left(\mathrm{T}_{2} \mathrm{w}\right)$, diffusion-weighted imaging (DWI), ultrashort-echo time imaging (UTE) and magnetisation transfer imaging (MT). Total protocol time: approx. $1 \mathrm{~h}$

\begin{tabular}{|c|c|c|c|c|}
\hline Modality & $\mathrm{T}_{2} \mathrm{~W}$ & DWI & UTE & MT \\
\hline Sequence type & TSE & 2D EPI & 3D mGRE & 3D GRE \\
\hline Slices & 24 & 18 & 96 & 30 \\
\hline FOV (mm) & $120 \times 72$ & $150 \times 105$ & $103 \times 103$ & $128 \times 96$ \\
\hline Slice thickness (mm) & 1 & 1.5 & 1.07 & 1 \\
\hline Matrix Size & $256 \times 152$ & $102 \times 72$ & $96 \times 96$ & $128 \times 96$ \\
\hline $\mathrm{TR}(\mathrm{ms})$ & 800 & 2100 & 42 & 15 \\
\hline $\mathrm{TE}(\mathrm{ms})$ & 9.6 & 60.8 & $7.16,11.64,16.12,20.60$ & 2.52 \\
\hline UTE (ms) & - & - & $0.07,0.14,0.28,0.56$ & - \\
\hline NSA & 1 & 18 & 1 & 8 \\
\hline iPAT & GRAPPA 2 & GRAPPA 2 & - & GRAPPA 2 \\
\hline Fat sat. & No & Yes & Yes & No \\
\hline $\mathrm{b}$-values $\left(\mathrm{mm}^{-2} \mathrm{~s}\right)$ & - & $\begin{array}{l}0,20,40,60,80,100 \\
200,400,800\end{array}$ & - & - \\
\hline Variable flip angles $\left({ }^{\circ}\right)$ & - & - & - & $4^{\circ}, 24^{\circ}$ \\
\hline MT pulse & - & - & - & without/with $(1.5 \mathrm{kHz})$ \\
\hline Time (min:s) & $4: 54$ & $15: 58$ & $4 \times 5: 36$ & $4 \times 2: 28$ \\
\hline
\end{tabular}


the observed $\mathrm{S}_{0}$ relative to the intercept of this curve at $\mathrm{b}=0$ $\mathrm{mm}^{-2} \mathrm{~s}$ :

$S_{b}-S_{o} \cdot \exp (-b . A D C)$

$S_{b}=S_{o} \cdot\left[f \cdot \exp \left(-b \cdot D^{*}\right)+(1-f) \cdot \exp (-b \cdot D)\right]$

where the observed signal intensity at a given b-value is denoted $\mathrm{S}_{\mathrm{b}}$, and $\mathrm{S}_{0}$ is the corresponding signal at $\mathrm{b}=0 \mathrm{~mm}^{-2} \mathrm{~s}$ (equal to the total available signal $S_{\text {total }}$ modulated by the apparent $\mathrm{T}_{2}$ and the acquisition echo time, $\mathrm{S}_{0}=\mathrm{S}_{\text {total }} \cdot \exp (-\mathrm{TE} /$ $\mathrm{T}_{\text {2app }}$ ) [23].

For UTE imaging, $\mathrm{T}_{2}{ }^{*}$ short was calculated using the first (ultrashort, < 1ms; see Table 1 for values) echo from successive imaging acquisitions, and the conventional $\mathrm{T}_{2}{ }^{*}$ long using the remaining (i.e. not ultrashort) echoes from all acquisitions, using separate mono-exponential models (Eq. 3); the ratio of the calculated signal arising, analogous to $f$ in the IVIM DWI model, from each of the two relaxation constants was also calculated. All DWI and UTE fitting was performed using a Markov Chain Monte Carlo (MCMC) Bayesian statistical approach [24] as a robust least-squares estimator, with no data filtering.

$S_{T E}=S_{o} \cdot \exp \left(-\frac{T E}{T_{2}^{*}}\right)$

MT acquisition images were used for calculation of magnetisation transfer ratio (MTR) (Eq. 4) [25, 26], longitudinal relaxation constants in the presence/absence of the MT pulse using the variable flip angle (VFA) method [27] $\left(\mathrm{T}_{1}\right.$ and $\mathrm{T}_{1 \mathrm{~s}}$, respectively), and $\mathrm{B}_{1}$-independent MT saturation $(\delta)$ and apparent MT rate $\left(\mathrm{k}_{\mathrm{a}}\right)$ (Eqs. 5 and 6) [26, 28]:

$M T R=\left(S_{r e f}-S_{M T}\right) / S_{r e f}$

$k_{a}=M T R / T_{1 s}$

$\delta=\left(R_{1 \text { app }} T R+\alpha_{\text {nom }}{ }^{2} / 2\right)\left(S_{\text {ref }}-S_{M T}\right) / S_{M T}$

where $\mathrm{S}_{\text {ref }}$ and $\mathrm{S}_{\mathrm{MT}}$ are signal amplitudes from identical sequences acquired with and without the MT pulse, TR is the acquisition repetition time, $R_{1 \text { app }}$ is the spin-lattice relaxation rate (or $\left.\mathrm{T}_{1}^{-1}\right), \alpha_{\text {nom }}$ is the nominal acquisition flip angle in radians, and the small flip angle approximation is used [26].

\section{Histological staining and analysis}

Following MR imaging, animals were killed by cervical dislocation, and the tumour excised and fixed in $10 \%$ formalin. Tumours were then cut through the centre, and embedded in paraffin blocks, with orientation matched to the geometry of the imaging slices to facilitate subsequent image correlation.

Tumour sections $(5 \mu \mathrm{m})$ were stained with haematoxylin and eosin (H\&E), to allow assessment of necrosis and tumour grade, and picrosirius red, to assess collagen I/III deposition (fibrosis). Immunohistochemistry visualised using DAB was performed using FITC-conjugated mouse monoclonal antibodies against pimonidazole adducts, followed by rabbit anti-FITC antibodies, for the detection of hypoxic regions, or rabbit monoclonal antibodies against CD31 (EP3095; Millipore, Watford, UK) to assess vascular endothelial cells as a proxy for perfusion. Whole tumour images were acquired using a motorised scanning stage (Prior Scientific Instruments, Cambridge, UK) attached to a BX51 microscope (Olympus Medical, Southend-on-Sea, UK) driven by CellP (Soft Imaging System, Munster, Germany). Snapshots at $\times 200$ magnification were also acquired from CD31-stained sections.

Tumour grade and degree of necrosis (semi-quantitative assessment) were evaluated by an expert pathologist (AN). Percentage area of each tumour section displaying pimonidazole adduct or picrosirius red positivity was measured using pixel counts from a customised routine operating on a Lab colour-space separation into stain and non-stain classes (Mathworks, Natick, MA, USA) of a digital image, and visually confirmed for accuracy. Microvessel density was assessed by counting CD31-positive vessels from six random fields $(\times 200)$ distributed across the section and the number converted to vessels $/ \mathrm{mm}^{2}$.

\section{Statistics}

MRI-derived parameters are given as the median of the ROI voxels in each observation/analysis, in order to minimise the contribution of outliers arising from partial volume effects. Correlations between MRI markers and histological analyses acquired from matched slices were assessed using Pearson correlation coefficients (r). Bonferroni correction for multiple comparisons against the different histological markers was applied, with results considered significant at $\mathrm{p}<0.0125$. A partial least-squares regression (PLSR) approach was applied to derived MR parameters for the response variable of picrosirius red stain, to assess the relative performance of a multiparametric approach. Leave-one-out cross-validation (LOOCV) was used to derive normalised root-mean-square error (NRMSE) as a proxy for goodness of response variable prediction.

\section{Results}

\section{Tumour cohort}

Tumours developed in a heterogeneous manner in the mammary fat pad of 15 rats, with imaging performed at an average tumour volume of $3.6 \pm 2.1 \mathrm{~cm}^{3}$ (average ROI slice area for 
analysis $338 \pm 168 \mathrm{~mm}^{2}$ ) over a wide timeframe post-injection of MNU (median 421 days, range 105-471). One animal simultaneously developed two tumours; both were imaged and analysed. Histology from two tumours was not satisfactorily matched to the imaging plane and was excluded from the analysis. A small sub-cohort $(n=4)$ of the largest tumours was sectioned in two places, into equally sized sections (> $5 \mathrm{~mm}$ thick), making 18 matched MR and histological data sets for analysis, from 14 tumours in 13 rats (one animal with two tumours, and two distinct regions each from four tumours).

\section{Histological slice matching and analysis}

Representative anatomical and functional images from two tumours are shown in Fig. 1, highlighting the varying contrast and resolution (including a typical ROI for analysis) obtained for each biomarker using the multiparametric MRI approach. The use of different slice thicknesses meant that the MR slice locations were not identical, but in each case were the closest match for the associated histology. Visual matching of the MRI with the corresponding histological sections was good, as demonstrated in Fig. 2 (same tumours as shown in Fig. 1).

Colour segmentation of picrosirius red staining and pimonidazole adduct immunohistochemistry successfully and robustly separated the desired stain from the remaining tissue and background (Supplementary Fig. S1), with repeated segmentation for picrosirius red staining giving essentially identical results (correlation 0.98, p > 0.0001). Details of the tumour cohort, including time from MNU injection to imaging (days) and tumour volume alongside histologically assessed tumour grade, degree of necrosis, microvessel density (MVD), percentage pimonidazole adduct formation and percentage picrosirius red staining, are given in Table 2.

\section{Repeatability of ROIs and MR parameters}

The Sørenson-Dice similarity coefficients of the ROIs drawn by the two observers ranged between 0.72 and 0.96 , with a median of 0.89 across all ROIs and no less than 0.88 within each MR modality, which demonstrates excellent agreement between observers. Repeat-measures percentage coefficients of variation (CoV) for positive-constrained MR-derived parameters were calculated using log-transformed values [29]. Excellent repeatability was shown for all MR parameters, with CoV values ranging from $1.5 \%$ to $8.6 \%$ (see Table 3 ), with the notable exception of IVIM $\mathrm{f}$ and $\mathrm{D}^{*}$, which are known to display poor repeatability [30].

\section{Diffusion-weighted imaging}

Scatter graphs of the diffusion parameters derived from both the ADC and IVIM models, plotted against the histological markers, are shown in Fig. 3 alongside example fitted parameter maps. A correlation of $r=0.801(p=0.0004)$ was found between the pseudodiffusion-related parameter $\mathrm{fD}^{*}$ and picrosirius red. Correlation of picrosirius red with the pseudodiffusion fraction $\mathrm{f}$ was non-significant under multiple comparison correction ( $\mathrm{r}=0.556, \mathrm{p}=0.0314)$. The IVIM D and monoexponential ADC parameters, notionally reporting on the same true diffusion phenomenon, were highly correlated $(\mathrm{r}=0.97, \mathrm{p}<0.001)$ as expected; both showed negative correlation with picrosirius red stain $(r=-0.574$ and -0.568 respectively; significance was not achieved under Bonferroni
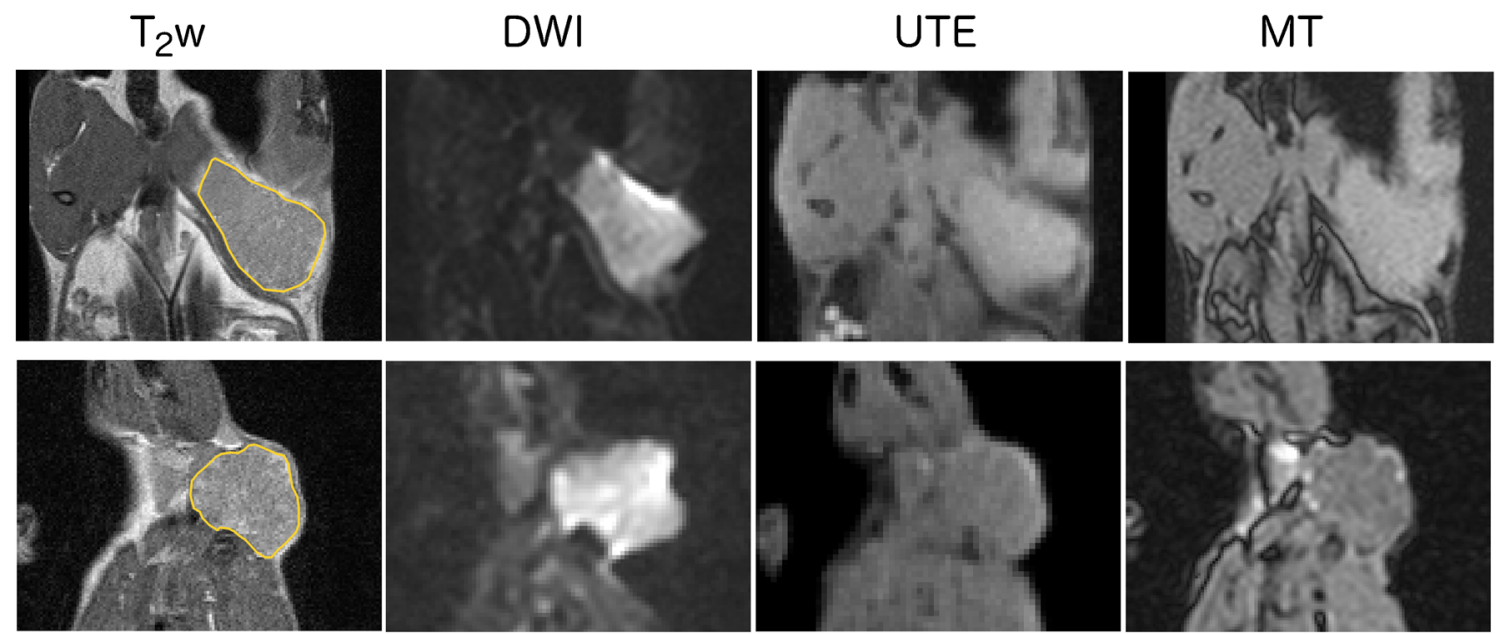

Fig. 1 Representative anatomical and functional images from two $\mathrm{N}$ methyl- $N$-nitrosourea-induced rat mammary carcinomas, showing the variation in tumour presentation and typical images from the multiparametric MRI strategy used herein: (a) $\mathrm{T}_{2}$-weighted

morphological imaging $\left(\mathrm{T}_{2} \mathrm{~W}\right)$, (b) diffusion-weighted imaging (DWI; $\mathrm{b}=0 \mathrm{~mm}^{-2} \mathrm{~s}$ ), (c) ultrashort-echo time imaging (UTE; TE $=0.07 \mathrm{~ms}$ ), and (d) magnetisation transfer imaging (MT; flip angle $4^{\circ}$, with MT pulse) 


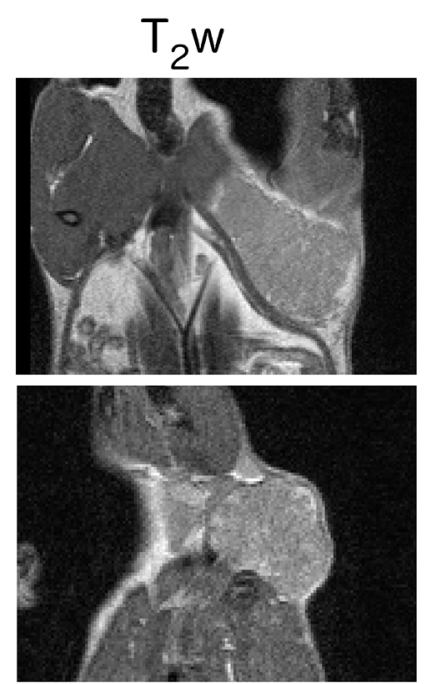

Fig. 2 Representative images from the same tumours as shown in Fig. 1 (upper row tumour 1, section 1, and lower row tumour 3 section 2; see Table 2 for analysis) showing matching of MRI with histology (left to

correction), but not with any other marker. IVIM pseudodiffusion parameters also correlated with percentage necrosis, though none were significant after multiple comparison correction ( $\mathrm{f}: \mathrm{r}=-0.607, \mathrm{p}=0.0165 ; \mathrm{D} * \mathrm{r}=0.556$, $\mathrm{p}=0.0313$; $\left.\mathrm{fD}^{*}: \mathrm{r}=-0.552, \mathrm{p}=0.033\right)$. The challenge of repeatably fitting pseudodiffusion parameters is reflected in the larger CoV. Correlation coefficients and $p$-values for

\section{Picrosirius Red Pimonidazole}
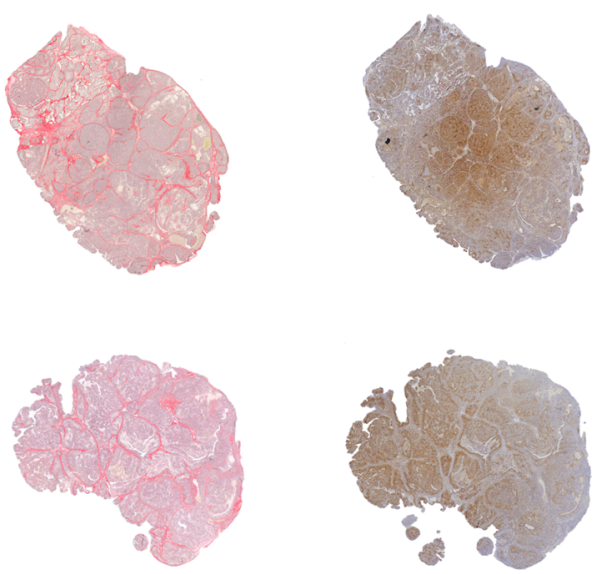

right) $\mathrm{T}_{2}$-weighted MRI, H\&E staining, picrosirius red staining for collagen I/III and pimonidazole adduct immunohistochemistry for hypoxia

comparisons of all MR parameters with histological markers are given in Table 3.

\section{Ultrashort-echo time imaging}

Typical parameter maps and data from UTE are shown in Fig. 4. There were no significant correlations with either CD31 or

Table 2 Cohort characteristics for animals with $N$-methyl- $N$-nitrosourea-induced mammary carcinoma

\begin{tabular}{|c|c|c|c|c|c|c|c|c|}
\hline Animal & Section & Days at scan & Volume $\left(\mathrm{cm}^{3}\right)$ & Tumour grade & Necrosis (\%) & $\begin{array}{l}\text { Microvessel density } \\
\left(\text { vessels } / \mathrm{mm}^{2}\right)\end{array}$ & Pimonidazole (\%) & Picrosirius red (\%) \\
\hline \multirow[t]{2}{*}{1} & 1 & 105 & 5.3 & 1 & 5 & 115 & 27.7 & 23.1 \\
\hline & 2 & & & 1 & 5 & 104 & 28.5 & 9.8 \\
\hline \multirow[t]{2}{*}{2} & 1 & 126 & 7.3 & 1 & 15 & 128 & 17.2 & 12.0 \\
\hline & 2 & & & 1 & 10 & 121 & 14.9 & 12.1 \\
\hline \multirow[t]{2}{*}{3} & 1 & 129 & 4.5 & 2 & 5 & 60 & 28.0 & 7.8 \\
\hline & 2 & & & 2 & 5 & 150 & 21.2 & 9.8 \\
\hline 4 & & 189 & 0.9 & 1 & 20 & 38 & 23.2 & 10.4 \\
\hline 6 & & 332 & 4.8 & 1 & $1-2$ & 65 & 23.2 & 52.3 \\
\hline 7 & & 372 & 2.6 & 3 & 5 & 114 & 19.9 & 16.8 \\
\hline \multirow[t]{2}{*}{8} & 1 & 421 & 3.6 & 1 & 0 & 68 & 32.1 & 28.1 \\
\hline & 2 & & & 1 & $1-2$ & 120 & 33.6 & 32.2 \\
\hline 10 & & 436 & 2.3 & 2 & 5 & 176 & 47.4 & 7.2 \\
\hline 11 & & 436 & 1.8 & 2 & 15 & 62 & 44.1 & 35.9 \\
\hline 12 & & 449 & 5.6 & 2 & $1-2$ & 100 & 21.6 & 32.8 \\
\hline $13-1$ & & 457 & 2.9 & 2 & 0 & 45 & 27.9 & 19.3 \\
\hline $13-2$ & & & 2.8 & 2 & 0 & 69 & 27.7 & 50.0 \\
\hline 14 & & 470 & 0.6 & 2 & 0 & 45 & 14.5 & 80.4 \\
\hline 15 & & 471 & 0.3 & 3 & 5 & 116 & 12.8 & 27.1 \\
\hline
\end{tabular}


Table 3 Correlation coefficients, $r$, between MR and histological parameters of matched slices

\begin{tabular}{|c|c|c|c|c|c|}
\hline \multirow[t]{2}{*}{ MR modality } & \multirow[t]{2}{*}{ MR parameter (repeatability $\mathrm{CoV}, \%$ ) } & \multicolumn{4}{|c|}{ Histological parameter ( $p$-value in parenthesis; bold indicates significance, $p<0.0125$ ) } \\
\hline & & Necrosis & MVD & Pimonidazole adduct & Picrosirius red \\
\hline \multirow[t]{5}{*}{ DWI } & $\operatorname{ADC}(6.14)$ & $0.328(0.232)$ & $0.185(0.5104)$ & $-0.078(0.7833)$ & $-0.568(0.0274)$ \\
\hline & $\mathrm{D}(5.18)$ & $0.309(0.2627)$ & $0.207(0.4587)$ & $-0.099(0.7244)$ & $-0.574(0.0253)$ \\
\hline & $\mathrm{f}(39.82)$ & $-0.607(0.0165)$ & $-0.269(0.3325)$ & $0.134(0.6349)$ & $0.556(0.0314)$ \\
\hline & $\mathrm{D}^{*}(58.42)$ & $0.556(0.0313)$ & $-0.070(0.8045)$ & $-0.172(0.5407)$ & $-0.035(0.9026)$ \\
\hline & $\mathrm{fD}^{*}(22.24)$ & $-0.552(0.033)$ & $-0.449(0.0933)$ & $0.071(0.8021)$ & $0.801(0.0004)$ \\
\hline \multirow[t]{3}{*}{ UTE } & $\mathrm{T}_{2}{ }^{*}$ long $(5.69)$ & $0.240(0.3701)$ & $0.409(0.1303)$ & $0.203(0.4501)$ & $-0.660(0.0055)$ \\
\hline & $\mathrm{T}_{2} *_{\text {short }}(8.58)$ & $-0.274(0.3038)$ & $0.305(0.269)$ & $0.085(0.7537)$ & $-0.092(0.734)$ \\
\hline & ratio (1.77) & $0.62(0.0105)$ & $0.291(0.2927)$ & $0.155(0.567)$ & $-0.290(0.2764)$ \\
\hline \multirow[t]{5}{*}{ MT } & MTR (1.57) & $-0.219(0.4142)$ & $-0.362(0.1851)$ & $0.06(0.8241)$ & $0.575(0.0198)$ \\
\hline & $\mathrm{T}_{1}(1.49)$ & $0.418(0.1075)$ & $0.377(0.1661)$ & $0.455(0.0763)$ & $-0.758(0.0007)$ \\
\hline & $\mathrm{T}_{1 \mathrm{~s}}(7.75)$ & $0.363(0.1673)$ & $0.363(0.1832)$ & $0.340(0.1981)$ & $-0.831(0.0001)$ \\
\hline & $\mathrm{k}_{\mathrm{a}}(7.88)$ & $-0.367(0.1623)$ & $-0.357(0.1912)$ & $-0.234(0.3831)$ & $0.857(0.0001)$ \\
\hline & $\delta(6.70)$ & $-0.521(0.0387)$ & $-0.537(0.0391)$ & $-0.209(0.4364)$ & $0.869(0.0001)$ \\
\hline
\end{tabular}

$A D C$ apparent diffusion coefficient, $M V D$ microvessel density, $D W I$ diffusion-weighted imaging, UTE ultrashort-echo time imaging, $M T$ magnetisation transfer imaging

pimonidazole adduct staining; significant correlation was observed only for $\mathrm{T}_{2}$ * long with picrosirius red $(\mathrm{r}=-0.660$, $\mathrm{p}=0.0055$ ).

\section{Magnetisation transfer imaging}

Correlations of the MT parameters with histological markers are shown in Fig. 5, alongside typical parameter maps. There were significant correlations for all MT parameters, excluding the $\mathrm{B}_{1}$-dependent measure MTR, with the percentage of picrosirius red staining. $T_{1}$ and $T_{1 \mathrm{~s}}$ showed similar negative correlations $(r=-0.758$ and 0.831 , respectively, $\mathrm{p}<0.001$ ), with decreased $\mathrm{T}_{1}$ correlated to increased picrosirius red stain. MTR had the weakest positive correlation $(r=0.575, p=0.0198)$, whereas accounting for $\mathrm{B}_{1}$-dependence in $\delta$ gave a stronger correlation ( $\mathrm{r}=0.869, \mathrm{p}=0.0001)$. The apparent MT rate constant $\mathrm{k}_{\mathrm{a}}$ was also significantly correlated with picrosirius red $(r=0.857, p=0.0001)$. CD31 and estimated necrosis also correlated with $\delta(\mathrm{r}=-0.537, \mathrm{p}=0.0391$ and $\mathrm{r}=-0.521$, $\mathrm{p}=0.0387$, respectively) but these were weaker and not significant following multiple comparison correction.

\section{Multiparametric partial least squares regression analysis}

The normalised root-mean-squared error (NRMSE) from LOOCV of linear regression for each of the MT parameters are presented in Table 4, and correspond to the observed correlations (Fig. 5). Conducting a PLSR analysis using all five MT parameters yielded a single-variable model, with loadings corresponding to the observed correlations, and a comparable NRMSE indicating that combinations of MT parameters do not necessarily outperform individual correlations. The corresponding PLSR using parameters across all MR modalities similarly gave a single LV model with a NRMSE that did not benefit from inclusion of other MR modalities, although DWI parameters ADC and D contributed to LV1. Loadings plots are shown in Supplementary Fig. S2 for both analyses.

\section{Discussion}

The presence of a histologically-confirmed fibrotic focus has been shown to be a predictor of increased tumour aggressiveness, relapse, metastasis and poor long-term survival in breast cancer [6-9]. Fibrotic foci are also associated with tumour hypoxia, an independent indicator of poor treatment response and prognosis [5, 18, 31-33]. The ability to detect fibrosis within mammary carcinomas non-invasively would be of great value in helping guide personalised treatment. The validation of appropriate MRI techniques with potential to inform on fibrosis using preclinical models with matched histology can directly guide development of imaging studies in the clinical setting.

In this study, a range of endogenous MR imaging contrasts were measured in chemically-induced mammary carcinomas arising in rats injected with MNU; the tumours were highly heterogeneous and presented with a range of fibrosis levels as previously observed in this model and typical of the clinical setting $[18,34]$. The imaging performed in the study used exclusively clinical hardware, conferring greater translational relevance to the study, and the scanning was performed within 

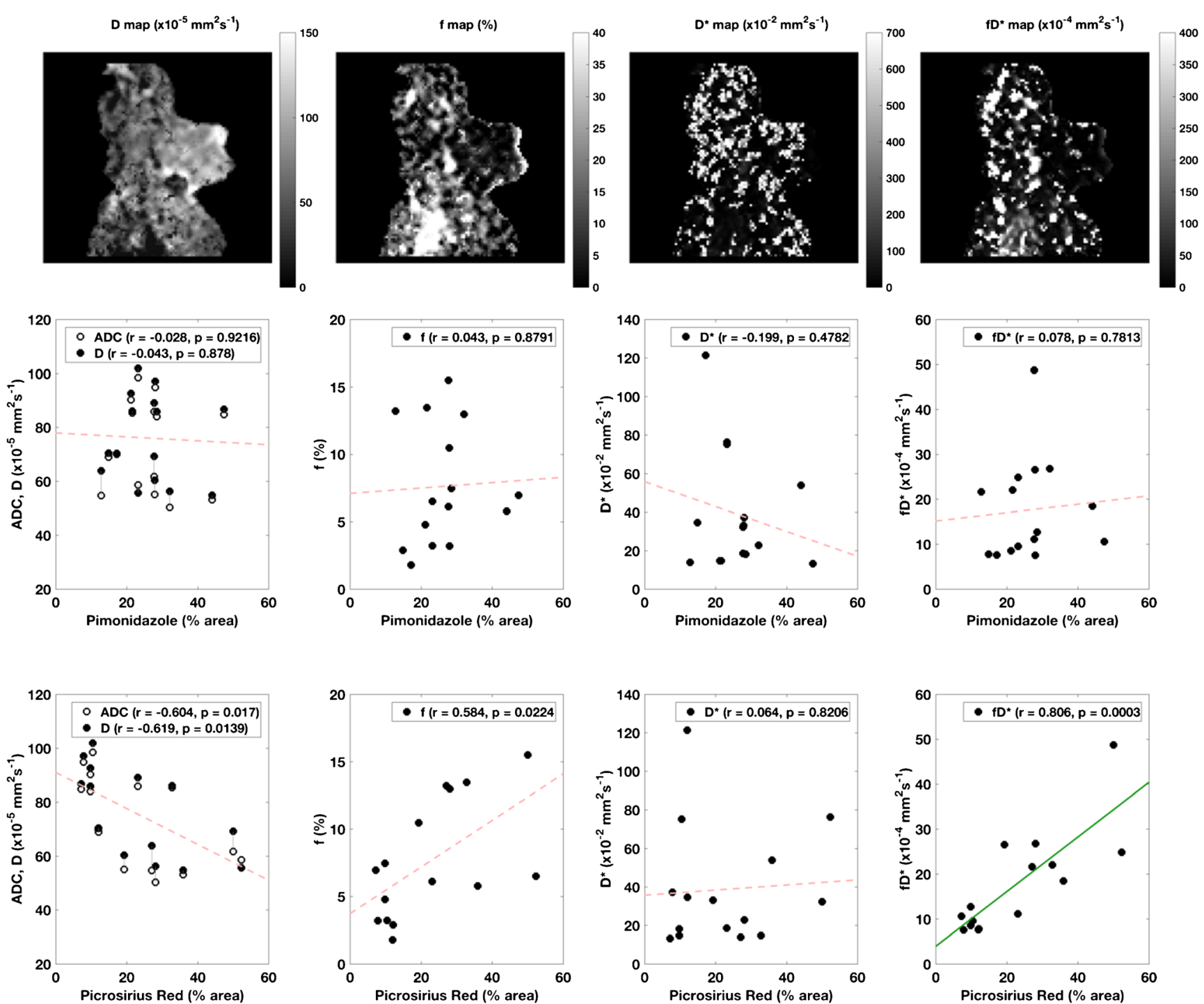

Fig. 3 Example maps (top row) for the fitted intravoxel incoherent motion (IVIM) functional parameters, with a binary mask to exclude pure noise, alongside scatter graphs of diffusion-weighted imaging parameters determined using the apparent diffusion coefficient (ADC) and IVIM models plotted against percentage pimonidazole adduct formation (middle row) and picrosirius red (bottom row) staining. Correlation

coefficients and $p$-values are given with each plot, with significant correlations (defined as $\mathrm{p}<0.0125$, corrected for multiple comparisons) found between IVIM fD* and picrosirius red (unbroken green lines). The combined plot for $\mathrm{ADC}$ and $\mathrm{D}$ indicates corresponding values; for clarity, only the regression line for $\mathrm{D}$ is shown

a clinical timeframe using standard and prototype (UTE and DWI) sequences developed by the manufacturer for use on the clinical platform. It has previously been shown that this platform is suitable for preclinical work of this nature [21,35], and can return functional MR parameters with good measurement repeatability across several imaging biomarkers. Repeated analysis by independent observers showed excellent repeatability of ROI positioning and all derived MR parameters except the pseudo-diffusion parameters from the IVIM diffusion model.

The results from the MT measurements were striking in their significance, with the presence of increased collagen leading to significant reductions in $T_{1}$ measurements, as well as increased $\mathrm{k}_{\mathrm{a}}$ and $\delta$. After correcting for multiple

comparisons, the correlations of these remained significant $(\mathrm{p}<0.0125)$. The MT ratio parameter, MTR, was correlated to picrosirius red stain fraction but fell short of significance. The similar parameter $\delta$, less dependent on the influence of $B_{1}$ [26], showed a stronger correlation and indicated that $B_{1}$ effects should be accounted for when analysing MT data. The fibrous macromolecule collagen has a much shorter spinlattice relaxation time $T_{1}$ compared to normal tissue, and through magnetisation transfer to water protons reduces the apparent $T_{1}$ of an imaging voxel dependent on the partial volume of collagen. The presence of the MT pulse saturates the collagen protons, and with transfer to the interacting water molecules, an additional and greater reduction occurs, giving much lower $\mathrm{T}_{1 \mathrm{~s}}$. The apparent MT rate constant for the 

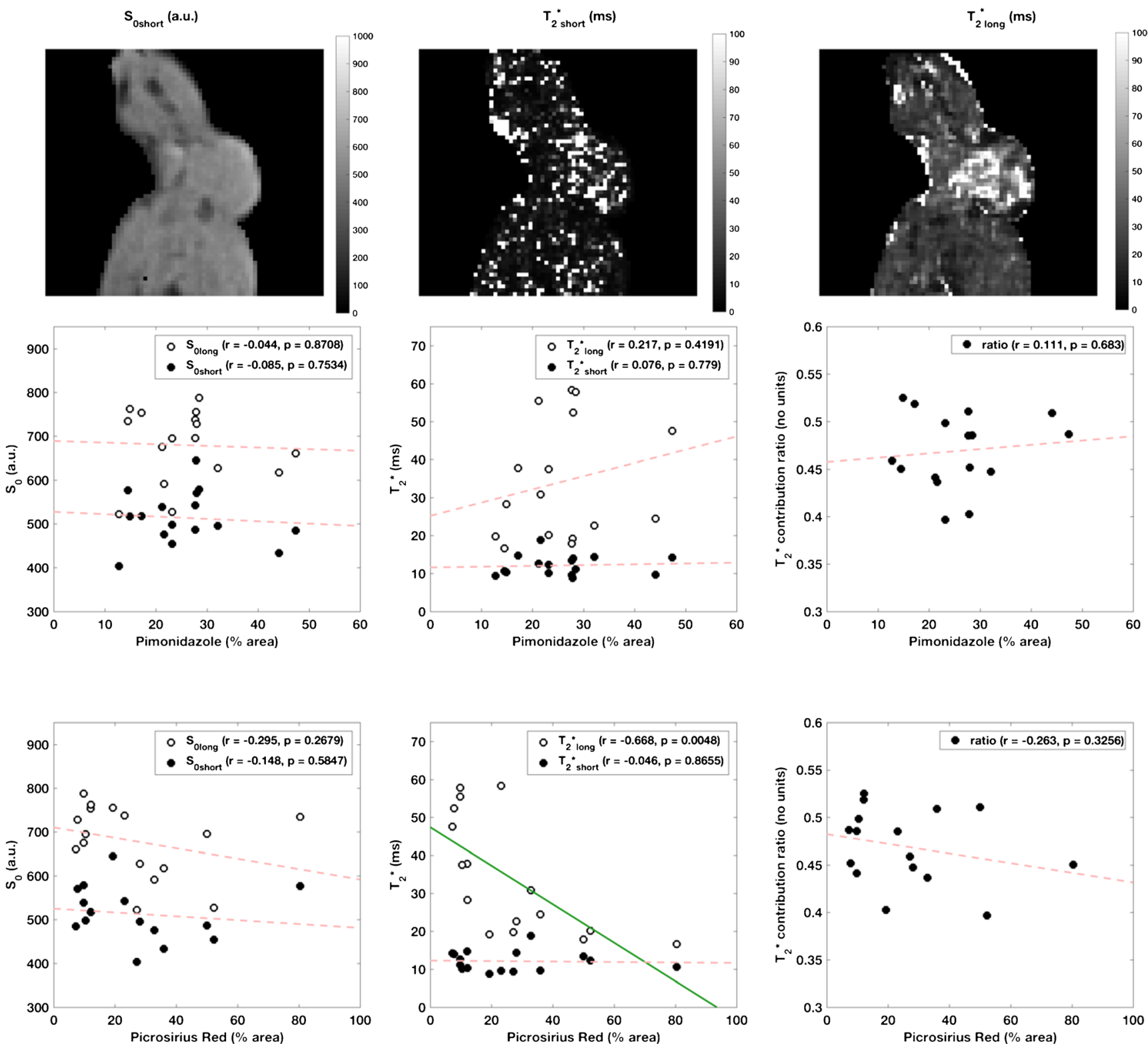

Fig. 4 Example maps (top row, with binary mask around animal) from fitting monoexponential model for $\mathrm{T}_{2} *$ using conventional $\left(\mathrm{T}_{2} *\right.$ long $)$ and ultrashort-echoes $\left(\mathrm{T}_{2} *\right.$ short $) . \mathrm{T}_{2} *$ parameters derived from ultrashort-echo time imaging, plotted against percentage pimonidazole adduct (middle row) and picrosirius red (bottom row) staining. Correlation coefficients and $p$-values are inset on each plot (significance defined as $\mathrm{p}<0.0125$, corrected for multiple comparisons, also indicated by an unbroken green regression line). The $\mathrm{T}_{2}{ }^{*}$ long, calculated from images with $\mathrm{TE}>7 \mathrm{~ms}$, shows correlation with picrosirius red staining destruction of the water signal by the MT saturation, $\mathrm{k}_{\mathrm{a}}$, is an empirical rather than a true rate constant [28], but does relate to the amount of collagen present, giving the observed correlation. Combining MT parameters using a PLSR analysis demonstrated a prediction error similar to that given from cross-validation using each parameter alone, indicating that different MT parameters provide statistically similar information on how collagen affects the tumour microenvironment. These results indicate that the MT measurement as performed was sensitive to the presence and proportion of collagen in the tumour, and can provide a non-invasive assessment of collagen content.

In diffusion-weighted imaging, the presence of collagen fibres will modify the diffusion characteristics of water molecules, providing additional barriers to free diffusion. In this study, the ADC and $\mathrm{D}$ values were negatively correlated with the picrosirius red staining, although with $p$-values short of significance $(\mathrm{p}=0.0274$ and 0.0253 , respectively), suggesting that the measurement of true diffusion is affected by the presence of fibrosis, in line with observations in hepatic fibrosis [36, 37]. These parameters were 

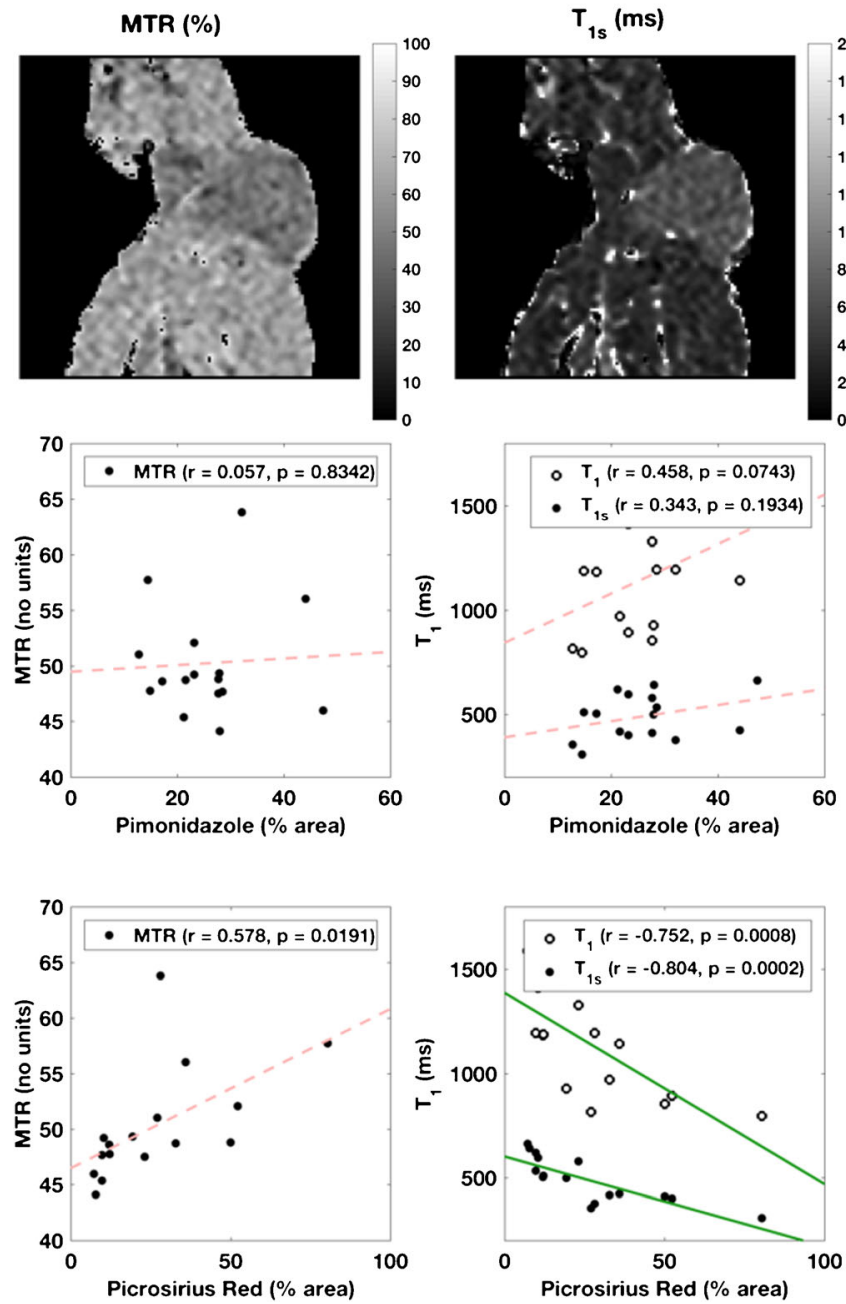

Fig. 5 Example magnetisation transfer parameter maps (top row, with binary mask around animal), with corresponding scatter plots with percentage pimonidazole adduct (middle row) and picrosirius red (bottom row) staining, including correlation coefficients and $p$-values

also found to contribute in the latent variables of the PLSR analysis, alongside MT parameters, although this model did not

Table 4 Comparison of single magnetisation transfer imaging (MT) parameters (1-5) with partial least squares regression analysis of (i) all MT, and (ii) all MR-derived parameters (latent variable details in Supplementary Fig. S2)

\begin{tabular}{lll}
\hline Variable & Model parameter & LOOCV NRMSE \\
\hline 1 & MTR & 0.3231 \\
2 & $\mathrm{~T}_{1}$ & 0.1930 \\
3 & $\mathrm{~T}_{1 \mathrm{~s}}$ & 0.1677 \\
4 & $\mathrm{k}_{\mathrm{a}}$ & 0.1663 \\
5 & $\delta$ & 0.1581 \\
All MT $(\mathrm{n}=5)$ & $\mathrm{LV} 1$ & 0.1731 \\
All MR $(\mathrm{n}=11)$ & LV1 & 0.2513
\end{tabular}

LOOCV leave-one-out cross-validation, NRMSE normalised root mean square error
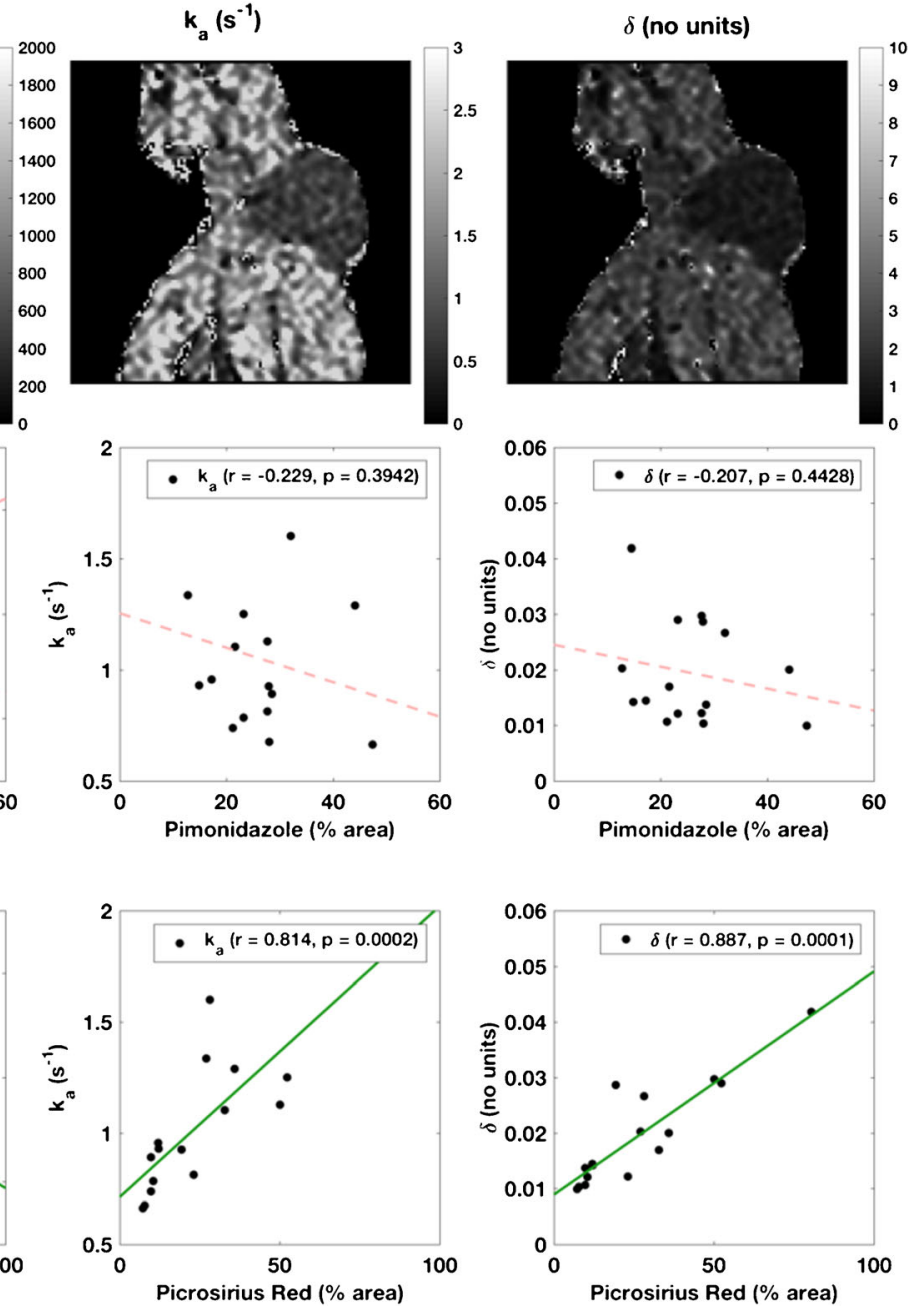

(inset). Significant $(\mathrm{p}<0.0125$, corrected for multiple comparisons) correlations were found for all magnetisation transfer (MT) parameters (unbroken green regression lines) except magnetisation transfer ratio (MTR) with percentage picrosirius red staining

outperform the best individual MT parameters. The fibrous nature of collagen may also introduce heterogeneity to the diffusion hindrance, manifesting as a non-Gaussian diffusion component captured as a significant positive correlation of collagen presence with the pseudo-diffusion parameter fD*. The data for the pseudo-diffusion volume fraction $\mathrm{f}$, often considered related to perfusion, showed no correlation with the endothelial marker CD31, which is likely reflective of the inherent difficulty in reliably fitting IVIM data, but also the complexity of tumour perfusion $[30,38]$. In contrast, the non-significant correlation of $\mathrm{f}$ with necrosis $(\mathrm{r}=-0.607, \mathrm{p}=0.0165$ ) may suggest that $\mathrm{f}$ does not solely capture vascular fraction [23] and may be related to the degree of non-Gaussian diffusion introduced by the presence of collagen fibres [14]. The high $\mathrm{CoV}$ values associated with the pseudodiffusion parameters, however, indicate that caution is required in interpreting these results.

In this study, the use of ultrashort echoes in order to visualise collagen did not give rise to a significant correlation. The 
conventional measurement of $\mathrm{T}_{2}{ }^{*}$ long, using echo times longer than the relaxation time of collagen, showed a correlation to picrosirius red, suggesting that the overall voxel $\mathrm{T}_{2} *$ is sensitive to the presence of fibrosis, and decreases with increasing collagen content.

The design of this study includes several limitations, which are nonetheless linked to its strengths. The use of clinical scanner hardware and imaging sequences means that while the scanner was not optimised for small animal studies, the techniques used were shown to be immediately translatable to clinical work. The mammary carcinoma model used in this work yielded tumours that varied considerably in presentation, growth rate, and composition; this reflects the clinical presentation of breast cancer and supports the potential of these results for clinical translation.

We have demonstrated the use of a multi-contrast MRI protocol to investigate the properties of chemically-induced mammary carcinoma in a preclinical setting, and have shown the potential of a clinical MT sequence to detect the presence of fibrosis noninvasively. Results from MT parameters outperformed those from multiple-b-value DWI and UTE imaging in detecting and quantifying intratumoral collagen, potentially providing information of biological relevance to support clinical assessment. Given that the presence of fibrosis is known to be a prognostic factor in mammary carcinoma, and may be induced following radiation therapy $[39,40]$, the results of this study support the inclusion of MT protocols in clinical breast MRI examinations.

Acknowledgements We thank Allan Thornhill and his staff for animal maintenance, and Dr. Berthold Kiefer of Siemens Healthcare, Erlangen, Germany, for provision of the prototype MR sequences used in this work. Thanks to Leslie Romelia Euceda Wood for helpful discussions on PLSR.

Funding This study has received funding by CRUK and EPSRC support to the Cancer Imaging Centre at The Institute of Cancer Research and The Royal Marsden Hospital in association with the MRC and Department of Health (England) (C1060/A10334, C1060/A16464), the CRUK and EPSRC Paediatric Imaging Programme grant (C7809/ A10342), and NHS funding to the NIHR Biomedical Research Centre and the Clinical Research Facility in Imaging at The Royal Marsden and the ICR. MOL is an NIHR Emeritus Senior Investigator.

\section{Compliance with ethical standards}

Guarantor The scientific guarantor of this publication is Simon P. Robinson.

Conflict of interest The authors of this manuscript declare no relationships with any companies whose products or services may be related to the subject matter of the article.

Statistics and biometry No complex statistical methods were necessary for this paper.

Ethical approval Approval from the institutional animal care committee was obtained.
Methodology

- prospective

- experimental

- performed at one institution

Open Access This article is distributed under the terms of the Creative Commons Attribution 4.0 International License (http:// creativecommons.org/licenses/by/4.0/), which permits unrestricted use, distribution, and reproduction in any medium, provided you give appropriate credit to the original author(s) and the source, provide a link to the Creative Commons license, and indicate if changes were made.

\section{References}

1. Egeblad M, Rasch MG, Weaver VM (2010) Dynamic interplay between the collagen scaffold and tumor evolution. Curr Opin Cell Biol 22:697-706

2. Cox TR, Erler JT (2011) Remodeling and homeostasis of the extracellular matrix: implications for fibrotic diseases and cancer. Dis Model Mech 4:165-178

3. Boyd NF, Martin LJ, Bronskill M et al (2010) Breast tissue composition and susceptibility to breast cancer. J Natl Cancer Inst 102: 1224-1237

4. Rønnov-Jessen L, Petersen O, Bissell M (1996) Cellular changes involved in conversion of normal to malignant breast: importance of the stromal reaction. Physiol Rev 76:69-125

5. Van Den Eynden GG, Colpaert CG, Couvelard A et al (2007) A fibrotic focus is a prognostic factor and a surrogate marker for hypoxia and (lymph)angiogenesis in breast cancer: review of the literature and proposal on the criteria of evaluation. Histopathology $51: 440-451$

6. Hasebe T, Tsuda H, Hirohashi S et al (1996) Fibrotic focus in invasive ductal carcinoma: an indicator of high tumor aggressiveness. Jpn J Cancer Res 87:385-394

7. Colpaert C, Vermeulen P, Jeuris W et al (2001) Early distant relapse in "node-negative" breast cancer patients is not predicted by occult axillary lymph node metastases, but by the features of the primary tumour 4082. J Pathol 193:442-449

8. Koyama T, Hasebe T, Tsuda H et al (1999) Histological Factors Associated with Initial Bone Metastasis of Invasive Ductal Carcinoma of the Breast. Jpn J Cancer Res 90:294-300

9. Hasebe T, Tsuda H, Hirohashi S et al (1998) Fibrotic focus in infiltrating ductal carcinoma of the breast: a significant histopathological prognostic parameter for predicting the long-term survival of the patients. Breast Cancer Res Treat 49:195-208

10. O'Connor JPB, Aboagye EO, Adams JE et al (2017) Imaging biomarker roadmap for cancer studies. Nat Rev Clin Oncol 14:169 186

11. Boult JK, Jamin Y, Jacobs V et al (2012) False-negative MRI biomarkers of tumour response to targeted cancer therapeutics. Br J Cancer 106:1960-1966

12. Luciani A, Vignaud A, Cavet M et al (2008) Liver Cirrhosis : Intravoxel Incoherent Motion MR Imaging — Pilot Study. Radiology 249:891-899

13. Watanabe H, Kanematsu M, Goshima S et al (2011) Staging Hepatic Fibrosis: Comparison of Gadoxetate Disodium-enhanced and Diffusion-weighted MR Imaging-Preliminary Observations. Radiology 259:142-150

14. Kakkad S, Zhang J, Akhbardeh A et al (2016) Collagen fibers mediate MRI-detected water diffusion and anisotropy in breast cancers. Neoplasia 18:585-593

15. Li W, Zhang Z, Nicolai J et al (2012) Magnetization transfer MRI in pancreatic cancer xenograft models. Magn Reson Med 68:12911297 
16. de Jong S, Zwanenburg JJ, Visser F et al (2011) Direct detection of myocardial fibrosis by MRI. J Mol Cell Cardiol 51:974-979

17. Bali MA, Metens T, Denolin V et al (2011) Tumoral and Nontumoral Pancreas: Correlation between Quantitative Dynamic Contrast-enhanced MR Imaging and Histopathologic Parameters. Radiology 261:456-466

18. McPhail LD, Robinson SP (2010) Intrinsic Susceptibility MR Imaging of Chemically Induced Rat Mammary Tumors: Relationship to Histologic Assessment of Hypoxia and Fibrosis. Radiology 254:110-118

19. Workman P, Aboagye EO, Balkwill F et al (2010) Guidelines for the welfare and use of animals in cancer research. Br J Cancer 102: $1555-1577$

20. Kilkenny C, Browne WJ, Cuthill IC et al (2010) Improving bioscience research reporting: The arrive guidelines for reporting animal research. PLoS Biol 8:e1000412

21. Jerome NP, Boult JKR, Orton MR et al (2016) Modulation of renal oxygenation and perfusion in rat kidney monitored by quantitative diffusion and blood oxygen level dependent magnetic resonance imaging on a clinical 1.5T platform. BMC Nephrol 17:142

22. Cho GY, Moy L, Zhang JL et al (2015) Comparison of fitting methods and b-value sampling strategies for intravoxel incoherent motion in breast cancer. Magn Reson Med 74:1077-1085

23. Jerome NP, D'Arcy JA, Feiweier T et al (2016) Extended T2-IVIM model for correction of TE dependence of pseudo-diffusion volume fraction in clinical diffusion-weighted magnetic resonance imaging. Phys Med Biol 61:N667-N680

24. Neil JJ, Bretthorst GL (1993) On the Use of Bayesian Probability Theory for Analysis of Exponential Decay Data: An Example Taken from Intravoxel Incoherent Motion Experiments. Magn Reson Med 29:642-647

25. Finelli DA, Reed DR (1998) Flip angle dependence of experimentally determined T1sat and apparent magnetization transfer rate constants. J Magn Reson Imaging 8:548-553

26. Helms G, Dathe H, Dechent P (2010) Modeling the influence of TR and excitation flip angle on the Magnetization Transfer Ratio (MTR) in human brain Obtained from 3D spoiled gradient echo MRI. Magn Reson Med 64:177-185

27. Wang HZ, Riederer SJ, Lee JN (1987) Optimizing the Precision in T1 Relaxation Estimation Using Limited Flip Angles. Magn Reson Med 5:399-416

28. Scholz TD, Ceckler TL, Balaban RS (1993) Magnetization transfer characterization of hypertensive cardiomyopathy: significance of tissue water content. Magn Reson Med 29:352-357
29. Jerome NP, Papoutsaki M-V, Orton MR et al (2016) Development of a temperature-controlled phantom for magnetic resonance quality assurance of diffusion, dynamic, and relaxometry measurements. Med Phys 43:2998-3007

30. Jerome NP, Miyazaki K, Collins DJ et al (2017) Repeatability of derived parameters from histograms following non-Gaussian diffusion modelling of diffusion-weighted imaging in a paediatric oncological cohort. Eur Radiol 27:345-353

31. Colpaert CG, Vermeulen PB, Fox SB et al (2003) The presence of a fibrotic focus in invasive breast carcinoma correlates with the expression of carbonic anhydrase IX and is a marker of hypoxia and poor prognosis. Breast Cancer Res Treat 81:137-147

32. Tatum JL (2006) Hypoxia: Importance in tumor biology, noninvasive measurement by imaging, and value of its measurement in the management of cancer therapy. Int J Radiat Biol 82:699-757

33. Goonewardene TI, Sowter HM, Harris AL (2002) Hypoxiainduced pathways in breast cancer. Microsc Res Tech 59:41-48

34. Williams J, Gusterson B, Humphreys J et al (1981) N-methyl-Nnitrosourea-induced rat mammary tumors. Hormone responsiveness but lack of spontaneous metastasis. J Natl Cancer Inst 66: $147-155$

35. Brockmann MA, Kemmling A, Groden C (2007) Current issues and perspectives in small rodent magnetic resonance imaging using clinical MRI scanners. Methods 43:79-87

36. Annet L, Peeters F, Abarca-Quinones J et al (2007) Assessment of diffusion-weighted MR imaging in liver fibrosis. J Magn Reson Imaging 25:122-128

37. Zhang Y, Jin N, Deng J et al (2013) Intra-voxel incoherent motion MRI in rodent model of diethylnitrosamine-induced liver fibrosis. Magn Reson Imaging 31:1017-1021

38. Jerome NP, Hekmatyar SK, Kauppinen RA (2014) Blood oxygenation level dependent, blood volume, and blood flow responses to carbogen and hypoxic hypoxia in 9L rat gliomas as measured by MRI. J Magn Reson Imaging 39:110-119

39. Wernicke AG, Rosenblatt R, Rasca M et al (2009) Quantitative assessment of radiation-induced fibrosis of the breast with tissue compliance meter, palpation, and radiological imaging: preliminary results. Breast J 15:583-592

40. Hammer C, Maduro JH, Bantema-Joppe EJ et al (2017) Radiationinduced fibrosis in the boost area after three-dimensional conformal radiotherapy with a simultaneous integrated boost technique for early-stage breast cancer: A multivariable prediction model. Radiother Oncol 122:45-49 